\title{
VentrAssist ${ }^{\mathrm{TM}}$ 人工心臓内流れの可視化
}

\author{
Flow Visualizations in VentrAssist ${ }^{\mathrm{TM}}$ Artificial Heart
}

\author{
○正 銭 逸（東覀大）
}

Yi QIAN, University of East Asia, 2-1 Ichnomiya-gakuenchu, Shimonoseki, Yamaguchi, 751-8503

\author{
Key Words: Flow Visualization, CFD, Blood pump, Verification and Validation.
}

\section{ABSTRACT}

The VentrAssist implantable Rotary Blood Pump (IRBP), which was named third general blood pump, and has been promoted to the stage of clinical pilot trial, has no shaft or seal, and the device is unique in design since the impeller of the pump is suspended within the pump diffuser passively by hydrodynamic forces. The functions of pump impeller and motor rotor are combined in the single moving part. The rotor is caused to rotate within the pump diffuser under the influence of a commutated electro-magnetic field provided by stator windings which are assembled just outside the pump diffuser. To this end, the flow is very difficult to be measured using usual laboratory equipments. Therefore, Computational Fluid Dynamics (CFD) has been applied as one of important tool in the VentrAssist ${ }^{\mathrm{M}}$ IRBP design and its validation procedure ${ }^{(1)}$. Several CFD results such as; pump performance improvement, unsteady hydraulic dynamic analysis, biocapability prediction, validation and verification (V\&V), and flow visualization have been performed.

In this work, flow visualization is used to investigate the hydrodynamic characteristics of the VentrAssist IRBP. Since the physical manifestation of the VentrAssist pump leaves no space for the insertion of devices into the pump for observing the flow field, it is not possible to determine the flow inside the pump using experimental flow visualization tools. Therefore, CFD becomes the only available method for observing the flow within the pump and hence is proposed in this study.

\section{INTRODUCTION}

It is well known that flow stagnation in blood can lead to thrombus formation. Thus, the washout characteristics are an important indicator of the antithrombogenetic properties of IRBP. Also, shear stress and residence time have been identified as major factors for destruction of the blood's red cells (haemolysis). Rotary blood pumps are required to work over a wide range of flow rates depending on the levels of physiological support needed. Moreover, the flow through the blood pumps usually has a pulsatile component which may have a significant effect on the flow patterns inside the pump. In general, recirculating flows set up may resist washout, prolong blood residence, and aggravate flow shear stress distributions. Therefore, flow visualizations are used to not only investigate the hydrodynamic characteristics of the Ventrassist ${ }^{\mathrm{TM}} \mathrm{IRBP}$, but also to predict its resulting biocompatibility.

CFD was applied to visual the flows through a VentrAssist ${ }^{\mathrm{TM}}$ IRBP. The three-dimensional flows through the pump were predicted and the flow patterns were visualized using CFD tools. The visualizations included two processes: flow simulation and flow observation. The flows were calculated using a commercial software; TASCflow, and observed using a CFD post-process tool; Fieldview. The visualizations were carried out at the pump design and off-design conditions under constant flow and Pulsatile flow conditions. Flow unsteady motion and flow washout capability have been shown by animation movers.

Although CFD simulations are widely employed in industry. CFD is a developing calculation procedure, so that there is a need to establish the uncertainty and error that may be associated with the modelling formulation and simulation procedures. Verification and validation $(\mathrm{V} \& \mathrm{~V})$ are the main principles used to determine the credibility of CFD results ${ }^{(2.3)}$.

\section{RESULTS}

\section{3-1 Verification and validation results}

Grid convergence was carried out. The grid number 227,705 (finial grid) insured a GCI smaller than 0.001 . This is a tight criterion as it is smaller than the value proposed by Roache ${ }^{(2)}$ for a much simpler flow. The subsystem simulation was validated by comparing numerical results with experimental results obtained using blood. The subsystem validation uncertainties were lower than $10 \%$ at all tested conditions.

\section{3-2 Flow simulation and visualization results}

Transient computational fluid dynamics results are able to be used to analyze the magnitude and direction of net force. and these results may provide a benchmark against which to check frozenrotor computations (which are much quicker to perform and which form the mainstay of all calculations carried out to date in the design of the VentrAssist ${ }^{\mathrm{TM}}$ IRBP). The steady CFD simulation cannot calculate a force peak between two maximum forces. It indicated that frozen-rotor steady CFD calculation cannot yield this type of information. In order to clearly visualize the flow washout capability, the visualizations were performed by postprocessing the results of CFD simulations. The flow paths through the pump were animated in the absolute frame of reference and visualized using the Fieldview.

\section{CONCLUSIONS}

Verification and Validation processes have been introduced to corroborate the CFD software for VentrAssist ${ }^{\mathrm{TM}}$ IRBP design and validation. The visualizations show that the VentrAssist ${ }^{\mathrm{TM}}$ IRBP exhibits a good washout environment, not only at the design point, but also at off-design conditions. No stable, attached stagnations are found within the pump. CFD studies provide a useful tool for visualizing the flow within rotary blood pumps and for assessing the potentials for thrombogenicity and haemolysis.

\section{REFERENCE}

1. Qian, Y., and Bertram, C., Computational fluid dynamics analysis of hydrodynamic bearing of the VentrAssist rotary blood pump, Artificial Organs, 24:488-91, 2000.

2. Roache, Patrick, J., Verification and Validation in Computational Science and Engineering, Hermosa Publishers, Albuquerque, New Mexico, USA, 1998

3. Anon, Guide for the Verification and Validation of Computational Fluid Dynamics Simulations, AIAA G-077-1998, 1998. 